\title{
DETERMINATION OF LUPEOL FROM MAKHAEN AT THAILAND AND TAIWAN BY HIGH- PERFORMANCE LIQUID CHROMATOGRAPHY
}

\section{SURACHAI TECHAOEI*, KHEMJIRA JARMKOM, NAKUNTWALAI WISIDSRI, SURADWADEE THUNGMUNGMEE, WARACHATE KHOBJAI}

Department of Thai Traditional Medicine College, Rajamangala University of Technology Thanyaburi, Pathum Thani, 12130 Thailand Email: Surachai_te@rmutt.ac.th

Received: 26 December 2018, Revised 29 December 2018 and Accepted: 16 February 2019

ABSTRACT

Objective: The objective of this research was to investigate the extraction and isolation of chemical compounds of Zanthoxylum limonella Alston extracts which selected from Chiang Mai and Pa Yao province, Thailand and Taiwan.

Method: There are 3 locations of medicinal plant, Z. limonella Alston was collected, two provinces of northern Thailand, Chiang Mai and Phayao, including the other country, Taiwan. All of extractions were carried out by methanol. In this experimental study, thin-layer chromatography (TLC) and high-performance liquid chromatography (HPLC) were measured.

Results: The result was found that the best organic solvent for extraction is methanol. The suitable of extraction was methanol and toluene in the ratio of 9:1 when developed with TLC and gradient methanol and water when analyzed with HPLC. The potential bioactive compound from crude extracted was lupeol. Crude extracted from leaf at Chiang Mai was highest lupeol as $5655.72 \mu \mathrm{g} / \mathrm{mg}$ extraction whereas crude extracted from leaf at Phayao showed this compound as $16,98.81 \mu \mathrm{g} / \mathrm{mg}$ extraction. In addition, the crude extraction from root and bark of both areas showed the amount of lupeol less than leaf crude extracted. The further study will focus on antimicrobial activity and biological activity in the near future.

Conclusion: The bioactive compound, lupeol was found in all part of Makhaen in Thailand but did not found in Taiwan seed.

Keywords: Zanthoxylum limonella Alston, Lupeol, Extraction, Medicinal plants.

(C) 2019 The Authors. Published by Innovare Academic Sciences Pvt Ltd. This is an open access article under the CC BY license (http://creativecommons. org/licenses/by/4. 0/) DOI: http://dx.doi.org/10.22159/ijap.2019.v11s5.T0045

\section{INTRODUCTION}

Zanthoxylum limonella Alston (Rutaceae) is medical plants, located in tropical and subtropical areas. In the past, this plant has been extensively used as a folk medicine for different medical purposes such as stomach ache, toothache, intestinal worms, rheumatism, scabies, snakebites, fever, and cholera [1]. The genus Zanthoxylum has been shown to produce a diverse range of secondary metabolites such as the alkaloid rutecarpine, coumarins, scopoletin, and xanthoxyletin, especially lupeol. Many compounds have been associated with different biologically activities, antimicrobial activity, anti-diarrheal, anticholinesterasic activity, antileishmanial activity, antiprotozoal activity, anti-larvicidal activity, and antioxidant activities [2].

In Thailand, Z. limonella Alston, locally called makhwaen, is widely distributed in the northern part of Thailand and has been traditionally used in food as spices. However, the reports on pharmacological properties and functional characteristic of Z. limonella have not been whole preformed in Thailand and South East Asia. In this study, we present data on the bioactive compounds of the crude extracts of Z. limonella Aston in Thailand compared with Chinese plant species at Taiwan.

\section{METHODS}

\section{Medicinal plant collection}

In this experimental study, there are 3 locations of medicinal plant, Z. limonella Alston was collected, two provinces of northern Thailand, Chiang Mai and Phayao, including the other country, Taiwan. These parts of plant material (Fig. 1) were washed under running tap water, air dried, and then homogenized to fine powder and stored in airtight bottles.
Medicinal plant extraction

All of extractions were carried out by $95 \%$ ethanol procedure as described by researcher [3]. $5 \mathrm{~g}$ of dried plants were extracted with $250 \mathrm{ml}$ of methanol and sonicated with $45^{\circ} \mathrm{C}$ for $30 \mathrm{~min}$. The process was repeated with the same solvent and pooled. The extracts were recovered from the solvent using rotary evaporator apparatus and stored in a freezer $\left(-20^{\circ} \mathrm{C}\right)$.

Thin-layer chromatography (TLC)

The extracts were checked qualitative analysis by TLC on analytical plates over silica gel. The crude extracts were applied on triplicate TLC plates, and toluene:methanol $(9: 1, \mathrm{v} / \mathrm{v})$ was used as developing solvent. After completion of run, the plates were removed from the chamber and allowed to dry in air. These plates were sprayed with freshly prepared $10 \%$ sulfuric acid and heated at 105 for $10 \mathrm{~min}$ for the presence of the spots, and the $R_{F}$ values of the spots were calculated from the ration of distance traveled by solute and distance traveled by solvent [4].

High-performance liquid chromatography (HPLC)

Sample solutions were thawed, filtered through $0.22 \mathrm{~mm}$ membrane filters, and then separated by reversed-phase HPLC (RP-HPLC) to obtain chromatograms. The amount of lupeol compounds was quantified using RP-HPLC at ambient temperature. The mobile phase consisted of $\mathrm{H}_{2} \mathrm{O}$ (solvent A) and methanol (solvent B). The flow rate was $1.0 \mathrm{ml} / \mathrm{min}$. The gradient program was set as follows in Table 1.

The injection volume was $500 \mu \mathrm{l}$, and the detect wavelength was $370 \mathrm{~nm}$. Analyses were performed in triplicate.

\section{Preparation of standard solutions}

Calibration curve for lupeol standard solutions was prepared at concentrations of $0.3125,0.625,1.25,2.5$, and $5 \mathrm{mg} / 5 \mathrm{ml}$ in methanol 
HPLC grade. Freshly prepared solutions were used for all determinations and were kept at $4^{\circ} \mathrm{C}$ and protected from light.

\section{Statistical analysis}

Data obtained were expressed as mean \pm standard deviation for groups $(n=3)$.

\section{RESULTS AND DISCUSSION}

\section{Extraction of medicinal plant}

The Z. limonella Aston. was extracted by ethanol solvent. Each crude extract was shown in term of percentage in Table 2 .

\section{Chromatographic separation by TLC}

The preliminary analysis to qualitatively analyze the presence of lupeol in the crude extract was performed using TLC on pre-coated silica gel $60 \mathrm{~F}_{254}$ plates. Using several developing solvents, the TLC method was applied to conduct the one which ensured the suitable condition of the difference bioactive molecules in the crude extract with special preference to lupeol. A standard lupeol solution was used as reference. Toluene and methanol in the ration as 9:1 was the solvent system proved to be the best condition as this resulted in the best separation of the components present in the crude extract.

In the subsequent purification steps, this system was used to check the presence of lupeol in the fraction taken. Part of the analysis was to choose the proper developing. After developing reagents, TLC plate was sprayed with $10 \%$ sulfuric acid in methanol solution (Fig. 2).

The active compounds and lupeol spot appeared on the TLC plate when heated at $120^{\circ} \mathrm{C}$ for $5-10 \mathrm{~min}$. In Fig. 2, the lupeol standard in lane 1 appeared pink in color with a $R_{F}$ value of 0.80 . The crude ethanolic extracts of Z. limonella Aston showed an intense band of lupeol at the same $R_{F}$ value as that of the standard.

\section{Linearity}

Calibration curve was constructed by plotting peak areas against concentration five lupeol standard solutions at $0.3125,0.625,1.25,2.5$, and $5 \mathrm{mg} / 5 \mathrm{ml}$ in methanol HPLC grade. Each sample was analyzed three times. A good linearity was obtained in the range evaluated (Fig. 3). The equations of calibration curve and regression coefficient were:

Table 1: The condition of high-performance liquid chromatography

\begin{tabular}{lllll}
\hline Time (min) & \% $\mathbf{M e O H}$ & $\mathbf{\% ~}_{\mathbf{2}} \mathbf{0}$ & $\mathbf{1 0 \%} \mathbf{M e O H}$ & $\mathbf{1 0 0 \%} \mathbf{M e O H}$ \\
\hline 0 & 0.0 & 0.0 & 80.0 & 20.0 \\
10 & 0.0 & 0.0 & 60.0 & 40.0 \\
20 & 0.0 & 0.0 & 50.0 & 50.0 \\
25 & 0.0 & 0.0 & 40.0 & 60.0 \\
30 & 0.0 & 0.0 & 30.0 & 70.0 \\
40 & 0.0 & 0.0 & 80.0 & 20.0 \\
45 & 0.0 & 0.0 & 80.0 & 20.0 \\
\hline
\end{tabular}

Table 2: The yield of crude extracts of Zanthoxylum limonella Aston

\begin{tabular}{lll}
\hline Part of plants & Appearance & Yield (\%) \\
\hline P1 & Green-brown, viscous & 0.92 \\
P2 & Green-brown, viscous & 2.69 \\
P3 & Green-brown, viscous & 2.36 \\
P4 & Green-brown, viscous & 1.10 \\
CM1 & Green-brown, viscous & 5.11 \\
CM2 & Green-brown, viscous & 4.22 \\
CM3 & Black, viscous & 1.97 \\
TW & Black, viscous & 2.67 \\
\hline
\end{tabular}

P1: Leaves at Phayao, P2: Bark at Phayao, P3: Root at Phayao, CM1: Bark at Chiang Mai, CM2: Leave at Chiang Mai, CM3: Leaves at Chiang Mai
Area $(Y)=151285 \times-129262$

Correlation coefficient $\left(\mathrm{R}^{2}\right)=0.997467$

The $\mathrm{R}^{2}$ obtained was higher than 0.99 , indicating a good linearity.

Determination of lupeol from Z. limonella Aston

The quantitative determination of lupeol using HPLC method mostly used acetonitrile/water as mobile phase. However, in this study, we selected methanol instead of acetonitrile because it was cheaper, not dangerous and produced the reasonable retention time. Peak area and
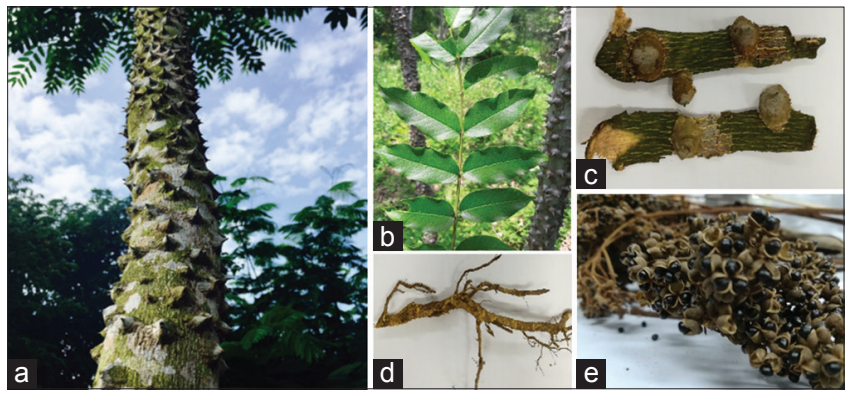

Fig. 1: The part of Zanthoxylum limonella Alston. (a) Stem (b) Leave (c) Bark (d) Root (e) Seeds

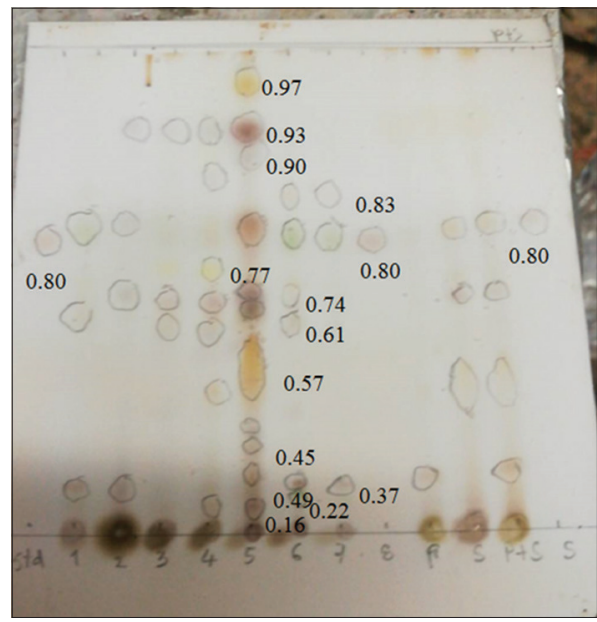

Fig. 2: Thin-layer chromatography chromatogram of Zanthoxylum limonella Alston extracts Std=lupeol standard, lane 1=P1, lane $2=P 2$, lane $3=P 3$, lane $4=P 3$, lane $5=C M 1$, lane 6-8=CM2,

$\mathbf{P}=$ pericarp Chiang Mai, $\mathbf{S}=$ seed Chiang $\mathrm{Mai}, \mathrm{P}+\mathrm{S}=$ pericarp and seed Chiang Mai

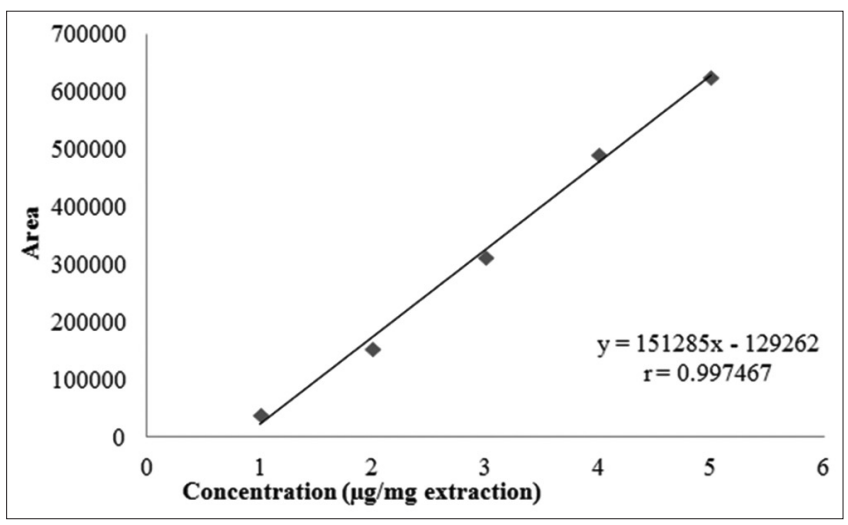

Fig. 3: The calibration curve obtained with lupeol standard solution $(n=3)$ 
Table 3: Peak area and retention time of Z. limonella Aston extracts

\begin{tabular}{lll}
\hline Part of plants & Retention time (s) & Lupeol $(\boldsymbol{\mu g} / \mathbf{m l})$ \\
\hline P1 & 9.77 & $5,655.729 \pm 1.13$ \\
P2 & 9.62 & $1,698.811 \pm 0.33$ \\
P3 & 9.65 & $1,414.857 \pm 0.28$ \\
P4 & 9.61 & $2,965.652 \pm 0.59$ \\
CM1 & 9.59 & $1,500.506 \pm 0.30$ \\
CM2 & 9.75 & $1,309.022 \pm 0.26$ \\
CM3 & 9.64 & $1,454.011 \pm 0.29$ \\
TW-S & ND & ND \\
TW-P & ND & ND \\
\hline
\end{tabular}

P1: Leaves at Phayao, P2: Bark at Phayao, P3: Root at Phayao, CM1: Bark at Chiang Mai, CM2: Leave at Chiang Mai, CM3: Leaves at Chiang Mai, ND: Not detected, TW-S: Z. limonella Aston seed extracts, TW-P: Z. limonella Aston pericarp extracts. Z. limonella: Zanthoxylum limonella

retention time of $Z$. limonella Aston extracts were shown in Table 3 . Thus, variation of environmental, ecological geographical conditions as well as culture conditions and extraction techniques results in a diversity of chemical components [5].

Some of species of the genus Zanthoxylum have been known to be used in traditional medicine for relieving pain. Previous phytochemical investigations on the different parts of Z. limonella showed quite wide variety of chemical substances. A number of secondary metabolites, alkaloids, aromatic and aliphatic amides, terpenes, sterols, and phenylpropanoid-lignans and coumarins has been isolated from this plant $[6,7]$. In addition, the lupeol compound was found to have antibacterial activity, anti-inflammatory, and anticancer $[8,9]$.

\section{CONCLUSION}

Z. limonella Aston is used as folk medicine and spices in food menu of any countries. The scientific literature shows the biological activity and phytochemically constituents. In these results showed the bioactive compound, lupeol which found in all parts of this species in Thailand but did not found in Taiwan species. Finally, the further study will focus on antimicrobial activity and biological activity such as anti-inflammatory effect and antioxidant activity in the near future.

\section{ACKNOWLEDGMENT}

The authors extend their appreciation to the Thai Traditional Medicine College, Rajamangala University of Technology Thanyaburi and Center of Excellence for Biodiversity, Chulalongkorn University for financial supported. The authors would like to thank Chinese herbal medicine laboratory, Department of Plant Industry, National Pingtung University of Science and Technology, Taiwan, especially Prof. Dr. Horng-Liang, Lay and Mr. Liao Shiu-Chi for HPLC characterization.

\section{REFERENCES}

1. Pierre MJ, Munyabuhoro M, Emmanuel BS. In vitro antimicrobial investigation of Zanthoxylum chalybeum stem bark. J Med Plants Res 2013;7:1157-79.

2. Majumder M, Sharma MH, Zaman K, Lyngdoh W. Evaluation of physic-chemical properties and antibacterial activity of the essentaial oil obtained from the fruits of Zanthoxylum acanthopodium DC. Collected from Meghalaya, India. Int J Pharm Sci 2014;6:543-6.

3. Janpen J, Roongtawan S, Warinthorn C. Antioxidant effect of Zanthoxylum limonella Alston. J Med Plants Res 2012;6:1407-14.

4. Patchanee C, Montinee T, Chamroon L. An allelopathic substrate isolated from Zanthoxylum limonella Alston fruit. Sci Hortic 2010;125:411-6.

5. Supabphol R, Tangitjareonkun J. Chemical constituents and biological activities of Zanthoxylum limonella (Rutaceae): A review. Trop J Pharm Res 2014;13:2119-30.

6. Simanek V. Benzophenanthridine alkaloids. In: Brossi A, editor. The Alkaloids, Chemistry and Pharmacology. London: Academic Press; 1985. p. 186-240.

7. Tangjitjaroenkun J, Chantarasriwong O, Chavasiri, W. Chemical constituents of the stems of Zanthoxylum limonella Alston. Phytochem Lett 2012;5:443-5.

8. Saleem M. Lupeol, a novel anti-inflammatory and anti-cancer dietary triterpene. Cancer Lett 2009;285:109-15.

9. Wongkattiya N, Akekawatchai C, Sanguansermsri P, Fraser IH, Pratoomsoot C, Sanguansermsri D. Chemical composition and biological properties of essential oils from Zanthoxylum rhetsa (Roxb.) DC and Zanthoxylum limonella Alston. Complement Altern Med $2018 ; 15: 12-8$ 